Revista Brasileira de Agricultura Irrigada v.10, nº.4, p. 715 - 725, 2016

ISSN 1982-7679 (On-line)

Fortaleza, CE, INOVAGRI - http://www.inovagri.org.br

DOI: $10.7127 /$ rbai.v10n400344

Protocolo 344.16 - 10/08/2015 Aprovado em 19/08/2016

\title{
AVALIAÇÃO DE MODELOS AGROMETEOROLÓGICOS QUE UTILIZAM A AMPLITUDE TÉRMICA DO AR ATMOSFÉRICO PARA A ESTIMATIVA DAS RADIAÇÕES GLOBAL E LÍQUIDA
}

\author{
José Geanini Peres ${ }^{1}$, Raquel Stucchi Boschi ${ }^{2}$, Claudinei Fonseca Souza ${ }^{1}$
}

\section{RESUMO}

Foram avaliados modelos agrometeorológicos para determinação das radiações global (Rs) e líquida (Rn) para a região de Araras-SP, o que foi feito a partir de informações medidas da temperatura máxima e mínima do ar atmosférico. Inicialmente, Rs e Rn foram estimadas por modelos consagrados na literatura, no caso da Rs pelos modelos de Hargreaves e Samani (MHS), Bristow e Campbell (MBC) e de Weiss (MWE); no caso da Rn pelos modelos de Zanetti (MZA) e Fietz e Fisch (MFF). Posteriormente, foram propostos modelos para a determinação de Rs e Rn específicos para a região de ArarasSP, a partir das informações de temperaturas máxima e mínima do ar atmosférico coletadas na estação meteorológica do Centro de Ciências Agrárias da UFSCar. Os modelos, existentes e propostos, foram avaliados quanto a sua exatidão e precisão por meio de análise de regressão linear, comparando-se os valores medidos de Rs e Rn com aqueles estimados pelos modelos. Dentre os modelos disponíveis na literatura, foi verificado que a Rs foi estimada com exatidão e precisão comparáveis pelos MHS, MBC e MWE, enquanto que a Rn foi estimada com excelente exatidão pelo MFF. Observouse, também, que a Rs e a Rn diárias para a região de Araras-SP podem ser estimadas pelos seguintes modelos potenciais: $\mathrm{Rs}_{\text {Inverno }}=0,041 \mathrm{Ra} \Delta \mathrm{T}^{\wedge 1,02}$, $\mathrm{Rs}_{\text {Verão }}=0,052 \mathrm{Ra} \Delta \mathrm{T}^{\wedge 0,91}$, $\mathrm{Rn}_{\text {Inverno }}=0,027 \mathrm{Ra} \Delta \mathrm{T}^{\wedge 0,96}$ e $\mathrm{R} n_{\text {Verão }}=0,040 \mathrm{Ra} \Delta \mathrm{T}^{\wedge 0,82}$, sendo Rs, Rn e Ra, respectivamente, as radiações global, líquida e extraterrestre $\left(\mathrm{MJ} \mathrm{m}^{-2} \mathrm{dia}^{-1}\right)$ e $\Delta \mathrm{T}$ a amplitude térmica diária do ar atmosférico $\left({ }^{\circ} \mathrm{C}\right)$.

Palavras-chave: balanço de radiação; radiação de ondas longas; radiação de ondas curtas.

\section{EVALUATION OF AGROMETEOROLOGICAL MODELS THAT USE THE THERMAL AMPLITUDE OF THE ATMOSPHERIC AIR IN THE ESTIMATION OF GLOBAL AND NET RADIATIONS.}

\footnotetext{
${ }^{1}$ Professor, Centro de Ciências Agrárias, UFSCar, Araras, SP, jogepe@cca.ufscar.br, cfsouza@cca.ufscar.br ${ }^{2}$ Pós-doutoranda, Escola Superior de Agricultura "Luiz de Queiroz", USP, Piracicaba, SP, raboschi@gmail.com
} 


\begin{abstract}
This study evaluated agrometeorological models for determination of global radiation (Rs) and net radiation ( $\mathrm{Rn}$ ) for the region of Araras-SP using measured information of maximum and minimum temperature of atmospheric air. Initially, Rs and Rn were estimated by models available in literature, in the case of Rs by Hargreaves and Samani (MHS), Bristow and Campbell (MBC) and Weiss (MWE), in the case of Rn by Zanetti (MZA) and Fietz and Fisch (MFF). Later, models were proposed for the determination of Rs and Rn specifically to the region of Araras-SP, also using information of maximum and minimum temperatures of atmospheric air collected at the weather station of the Center for Agricultural Sciences of UFSCar. The models, available and proposed, were evaluated about its accuracy and precision by linear regression analysis, comparing the measured values of Rs and Rn with those estimated by the models. Among the models available in the literature, it was found that Rs was estimated with accuracy and precision comparable by MHS, MBC and MWE, while Rn was estimated with excellent accuracy by MFF. It was observed that the Rs and Rn daily values for the region Araras-SP can be estimated by the following potential models: Rs Inverno $=0.041 \mathrm{Ra} \Delta \mathrm{T}^{\wedge} 1.02$, Rsverão $=0.052$ Ra $\Delta \mathrm{T}^{\wedge} 0.91, \mathrm{Rn}_{\text {Inverno }}=0.027 \mathrm{Ra} \Delta \mathrm{T}^{\wedge} 0,96$ and $\mathrm{Rn}_{\text {Verão }}=\mathrm{Ra} \Delta \mathrm{T}^{\wedge} 0,82$, being $\mathrm{Rs}, \mathrm{Rn}$ and $\mathrm{Ra}$, respectively, global, net and extraterrestrial radiations $\left(\mathrm{MJ} \mathrm{m}^{-2}\right.$ day $\left.^{-1}\right)$ and $\Delta \mathrm{T}$ the daily thermal amplitude of atmospheric air $\left({ }^{\circ} \mathrm{C}\right)$.
\end{abstract}

Keywords: radiation balance; longwave radiation, shortwave radiation.

\section{INTRODUÇÃO}

A radiação global é uma variável meteorológica muito importante para a caracterização climática de uma região, uma vez que ela caracteriza a quantidade de energia disponível para o crescimento das plantas. Apesar da determinação da quantidade de radiação global no nível do solo ser fundamental para o desenvolvimento de projetos de aproveitamento da energia solar em suas várias aplicações, sua utilização fica prejudicada pela dificuldade da sua medição (Khatib et al., 2012).

A radiação global pode ser medida utilizando-se diferentes equipamentos, destacando-se, o piranômetro nas estações meteorológicas automatizadas e o actinógrafo nas estações meteorológicas convencionais. Como são equipamentos sensíveis e caros, que demandam manutenção periódica e especializada, a sua utilização no Brasil está restrita basicamente aos institutos de pesquisa e às universidades públicas.

Tradicionalmente, a radiação global é estimada através do modelo de AngstromPrescott, que a relaciona com a radiação solar no topo da atmosfera terrestre e com a razão de insolação (Pereira, et al., 2002; Allen et al., 1998; Burman e Pochop, 1994; Doorenbos e Pruitt, 1977). A grande dificuldade para a aplicação deste modelo reside na determinação da duração do brilho solar, pois o equipamento utilizado na sua medição, normalmente um heliógrafo do tipo Campbell-Stokes, nem sempre está disponível localmente.

Para superar esta dificuldade, modelos empíricos forma desenvolvidos para estimativa da radiação global a partir de variáveis meteorológicas de fácil obtenção, tais como, temperatura do ar (Hargreaves e Samani, 1982; Bristow e Campbell, 1984; Goodin et al., 1999; Benghanem et al., 2014; Quej et al., 2016), nebulosidade (Badescu e Dumitrescu, 2013), duração do brilho solar (Al-Mostafa et al., 2014; Despotovis et al., 2015) e precipitação (Liu e Scott, 2001; Kirmani et al., 2015). Dentre as variáveis, a temperatura do ar tem apresentado bons resultados na estimativa da radiação solar (Weiss et al., 2001; Liu et al., 2009; Khatib et al., 2012; Yacef et al., 2014; Santos et al., 2015), além de ser a variável de mais fácil disponibilidade.

Já a determinação da radiação líquida é mais difícil do que a da radiação global devido 

AR ATMOSFÉRICO PARA A ESTIMATIVA DAS RADIAÇÕES GLOBAL E LÍQUIDA

às variações que ocorrem no albedo das superfícies (Burman e Pochop, 1994). Sendo assim, a aplicação de modelos baseados na radiação solar global e em variáveis meteorológicas de fácil aquisição, como a temperatura e a umidade relativa do ar atmosférico, é prática comum para determinação da radiação líquida (Zanetti et al., 2006; Fietz e Fisch, 2008; Montone e Sentelhas, 2010; Sentelhas e Nascimento, 2003).

O presente trabalho de pesquisa teve como objetivo avaliar e propor modelos para a determinação das radiações global (Rs) e líquida (Rn) para a região de Araras-SP a partir de informações medidas das temperaturas máxima e mínima do ar atmosférico. Em uma primeira etapa, Rs e Rn foram estimadas utilizando-se modelos disponíveis na literatura; posteriormente, numa segunda etapa, foram propostos modelos para a estimativa de Rs e Rn especificamente para a região de Araras-SP.

\section{MATERIAL E MÉTODOS}

\section{Área de estudo e Conjunto de dados}

As informações meteorológicas utilizadas nesta pesquisa - radiação global, radiação líquida, temperatura máxima e temperatura mínima - foram coletadas durante os anos de 2008, 2009, 2010 e 2011 na estação meteorológica automática do Centro de Ciências Agrárias, da Universidade Federal de São Carlos (CCA-UFSCar), que fica localizado no município de Araras-SP. O CCA-UFSCar está localizado a $22^{\circ} 18^{\prime}$ de latitude sul e $47^{\circ} 23^{\prime}$ de longitude oeste, e altitude média de aproximadamente $700 \mathrm{~m}$.

As normais climatológicas anuais locais são as seguintes: precipitação pluvial de 1.414 $\mathrm{mm}$; temperatura média do ar de $21,1{ }^{\circ} \mathrm{C}$; evaporação do tanque Classe A: $1443 \mathrm{~mm}$; velocidade média do vento: $1,44 \mathrm{~m} / \mathrm{s}$; umidade relativa do ar: 68,6 \%; insolação: 2.573 horas. $\mathrm{O}$ clima da região, segundo a classificação de Koppen, é do tipo Cwa, com verões quentes e úmidos e invernos secos.

Na coleta das informações meteorológicas foram utilizados um sensor de temperatura e umidade relativa, modelo HMP35C; um sensor de radiação global, modelo LI200X, e um sensor de radiação solar líquida, modelo Q7.1, espectro de 0,25 a $60 \mu \mathrm{m}$.

Todas as informações meteorológicas foram processadas e agrupadas para formarem três conjuntos de dados meteorológicos. O primeiro grupo foi formado grupando-se todos os valores diários válidos coletados nos períodos analisados, recebendo a denominação de Total. Um segundo conjunto foi formado grupando-se as informações coletadas na primavera e no verão, que recebeu o nome genérico de Verão. Por último, as informações coletadas nos meses de outono e inverno foram grupadas formando o conjunto de dados meteorológicos denominado de Inverno.

A radiação solar extraterrestre $(\mathrm{Ra})$ foi estimada diariamente seguindo os procedimentos propostos por Allen et al. (1998).

\section{Modelos baseados na temperatura do ar para estimativa da Radiação Global e Líquida}

A radiação global (Rs) foi estimada inicialmente pelos modelos de Hargreaves e Samani (1982) (MHS), de Bristow e Campbell (1984) (MBC), e de Weiss et al. (2001) (MWE). O modelo de Hargreaves e Samani (1982) utiliza para a determinação da Rs informações tabeladas da radiação solar extraterrestre e da raiz quadrada da amplitude térmica diária, ou seja, o modelo de ajustamento é uma função potência, no caso uma função raiz. O modelo de Bristow e Campbell (1984) utiliza os mesmos parâmetros climáticos daquele proposto por Hargreaves e Samani, dele desviando apenas no tipo de função utilizada no ajustamento das informações medidas e estimadas. O modelo de Weiss et al. (2001) incorpora em sua estrutura matemática informações sobre a Ra e uma função potência do segundo grau da amplitude térmica. O modelo assim obtido guarda uma grande semelhança com o modelo de Bristow e Campbell.

A radiação líquida $(\mathrm{Rn})$ foi estimada pelos modelos de Zanetti et al. (2006) (MZA) e de Fietz e Fisch (2008) (MFF). O modelo proposto por Zanetti et al (2006) utiliza como variáveis 
independentes a Ra e a amplitude térmica do ar atmosférico. Fietz e Fisch (2008) utilizam dados de temperatura do ar, da radiação extraterrestre e da Rs.

Os modelos propostos para a estimativa de Rs e $\mathrm{Rn}$ para a região de Araras-SP foram obtidos correlacionando-se a razão entre a Rs e a Ra (Rs/Ra) com a amplitude térmica diária do ar atmosférico $(\Delta \mathrm{T}=\mathrm{Tmax}$-Tmin $)$ As informações climatológicas utilizadas nestas determinações foram coletadas nos anos de 2009 e 2010, enquanto que na validação destes modelos foram utilizadas as informações coletadas durante os anos de 2008 e 2011.

No estabelecimento dos modelos propostos para a estimativa das radiações global e líquida para a região de Araras-SP foram testadas as relações funcionais expressas nas Equações de 1 a 6 .

$$
\begin{aligned}
& \frac{R s}{R a}=f(T \max -T \text { min }) \\
& \frac{R n}{R a}=f(T \max -T \text { min }) \\
& \frac{R s}{R a}=f(T \max -T \min )^{1 / 2} \\
& \frac{R n}{R a}=f(T \max -T \min )^{1 / 2} \\
& \frac{R s}{R a}=f\left(\exp ^{T \max -T \min ^{1 / 2}}\right) \\
& \frac{R n}{R a}=f\left(\exp ^{T \max -T \min ^{1 / 2}}\right)
\end{aligned}
$$

nas quais o termo Rs é a radiação global (MJ m $\left.{ }^{2} \mathrm{dia}^{-1}\right)$; Rn a radiação líquida $\left(\mathrm{MJ} \mathrm{m}^{-2} \mathrm{dia}^{-1}\right)$; Ra a radiação extraterrestre $\left(\mathrm{MJ} \mathrm{m}^{-2} \mathrm{dia}^{-1}\right)$; Tmax a temperatura máxima diária do ar $\left({ }^{\circ} \mathrm{C}\right)$ e Tmin a temperatura mínima diária do ar $\left({ }^{\circ} \mathrm{C}\right)$.

É importante destacar que nas Equações (1) a (6) a diferença de temperatura do ar atmosférico (Tmax-Tmin) corresponde a amplitude térmica diária do ar, em ${ }^{\circ} \mathrm{C}$, neste texto representada por $\Delta \mathrm{T}$, tal que $\Delta \mathrm{T}=($ Tmax-Tmin $)$.

Os modelos empíricos disponíveis na literatura utilizados e os que foram propostos nesta pesquisa para a estimativa da Rs e da Rn foram avaliados quanto ao seu desempenho - exatidão e precisão - por meio de análise de regressão, na qual suas estimativas foram comparadas com os valores medidos na estação meteorológica do CCAUFSCar.

As análises de regressão foram realizadas tomando-se os valores medidos da Rs e da Rn como variáveis dependentes e as suas estimativas pelos modelos empíricos, os disponíveis na literatura e os desenvolvidos nesta pesquisa, como variáveis independentes. O modelo de melhor desempenho nas estimativas de Rs e Rn foi aquele que apresentou coeficiente de determinação $\left(\mathrm{R}^{2}\right)$ mais alto e coeficiente angular (b) mais próximo de 1.

\section{RESULTADOS E DISCUSSÃO}

Na Tabela 1 estão apresentados os valores de coeficiente angular do modelo (b) e respectivo coeficiente de determinação $\left(\mathrm{R}^{2}\right)$ obtidos a partir das análises de regressão entre os valores medidos e estimados da radiação global em Araras-SP por meio dos modelos de Hargreaves e Samani (MHS), Bristow e Campbell (MBC) e Weiss (MWE).

Tabela 1. Valores do coeficiente angular do modelo (b) e do coeficiente de determinação $\left(\mathrm{R}^{2}\right)$ utilizados na avaliação do desempenho dos modelos Hargreaves e Samani (MHS), Bristow e Campbell (MBC) e Weiss (MWE) na estimativa da radiação global (Rs) em Araras-SP.

\begin{tabular}{ccccccccc}
\hline Modelo & \multicolumn{2}{c}{ Total } & \multicolumn{2}{c}{ Verão } & \multicolumn{2}{c}{ Inverno } & \multicolumn{2}{c}{ Média } \\
\cline { 2 - 10 } & $\mathrm{b}$ & $\mathrm{R}^{2}$ & $\mathrm{~b}$ & $\mathrm{R}^{2}$ & $\mathrm{~b}$ & $\mathrm{R}^{2}$ & $\mathrm{~b}$ & $\mathrm{R}^{2}$ \\
\hline
\end{tabular}


AVALIAÇÃO DE MODELOS AGROMETEOROLÓGICOS QUE UTILIZAM A AMPLITUDE TÉRMICA DO AR ATMOSFÉRICO PARA A ESTIMATIVA DAS RADIAÇÕES GLOBAL E LÍQUIDA

\begin{tabular}{lllllllll}
\hline MHS & 0,96 & 0,57 & 1,30 & 0,54 & 1,19 & 0,66 & 1,15 & 0,59 \\
MBC & 0,81 & 0,61 & 0,71 & 0,62 & 0,93 & 0,69 & 0,82 & 0,64 \\
MWE & 0,85 & 0,55 & 0,76 & 0,53 & 0,96 & 0,69 & 0,86 & 0,59
\end{tabular}

Um ponto importante que pode ser observado na Tabela 1 é que todos os modelos testados estimaram a Rs com exatidão mais alta no grupamento Inverno do que no de Verão. Esta particularidade, que ratifica as disposições de Allen et al. (1998) e Bristol e Campbell (1984), pode ser explicada pela ocorrência de um número menor de dias nublados nos meses do Inverno do que nos do Verão, cujo resultado é uma correlação mais estreita entre a Rs disponível e a temperatura do ar ao nível do solo.

Quanto à exatidão das estimativas da radiação global, aqui medida pelos coeficientes angulares das equações de regressão (b), pode ser verificado que o MHS teve melhor desempenho do que os outros dois modelos no caso do grupamento Total. Neste mesmo grupamento de dados, pode ser observado, ainda, que o erro cometido na estimativa da radiação global pelo MHS foi de apenas 4\%, enquanto que, no caso do MBC e do MWE, o erro comentido foi mais elevado, respectivamente, de 19 e 15\%. No caso do grupamento de dados Verão, pode ser observado que os erros cometidos na estimativa da radiação global foram praticamente os mesmos para os três modelos (MHS: 30\%; MBC: 29\% e MWE: $24 \%$ ). No caso do grupamento Inverno, o MBC teve um desempenho ligeiramente superior ao MWE, erros de $4 \%$ no primeiro modelo e de $7 \%$ no segundo; enquanto que o erro cometido pelo MHS foi bem mais elevado, de $19 \%$.

Quanto à precisão dos modelos, aqui medida pelos coeficientes de determinação $\left(R^{2}\right)$ das equações de regressão, pode ser verificado ela foi aproximadamente a mesma para os três modelos estudados, com ligeira vantagem para o MBC, considerando-se nesta avaliação os três grupamentos de dados meteorológicos adotados neste estudo.
Resumindo o que foi exposto, pode ser concluído que, em valores absolutos, os erros médios cometidos pelos três modelos na estimativa da radiação solar foram praticamente os mesmos, ou seja, $15 \%$ no caso do MHS e erros de $18 \%$ e $14 \%$, respectivamente, para o MBC e MWE. Conceição e Marin (2007), testando o modelo de MHS e de MBC nas condições edafoclimáticas de Jales-SP, Campinas-SP, Petrolina-PE e Teresina-PI, verificaram que a Rs foi estimada com grande exatidão pelo modelo. O MHS superestimou a radiação global em apenas $2 \%$, portanto um erro muito pequeno (coeficiente angular médio entre valor medido e estimado de 0,98). Quanto à precisão do modelo o $\mathrm{R}^{2}$ médio foi de 0,61 . O MBC subestimou a radiação global em apenas $2 \%$, um erro muito pequeno (coeficiente angular médio entre valor medido e estimado de 1,02). Já à precisão do modelo foi superior a obtida pelo MHS, com um coeficiente de determinação $\left(\mathrm{R}^{2}\right)$ médio de 0,70. A utilização dos modelos MHS e MBC para estimativa da Rs também foi realizada com sucesso por Conceição e Mandelli (2005) e por Conceição (2009). Quej et al. (2016) testaram 13 modelos para diferentes locais da península de Yucatán no México, dentre eles o MHS e o MBC. Para o MHS o erro médio variou de $-0,35$ a 0,63 e um $\mathrm{R}^{2}$ entre 0,49 e 0,62. Já o MBC superestimou os dados para todas as regiões, sendo os desvios menores quando comparados ao MHS, com valores de erro médio entre 0,08 e 0,58 e $\mathrm{R}^{2}$ variando de 0,49 a 0,63. O MWE foi testado por Borges et al. (2010) nas condições edafoclimáticas de Cruz das Almas-BA. Os autores verificaram que este modelo apresentou baixa exatidão na determinação da Rs, subestimando-a, em média, em $68 \%$, com um $\mathrm{R}^{2}$ de 0,72 .

Na Tabela 2 estão apresentados os valores de coeficiente angular do modelo (b) e 
respectivo coeficiente de determinação $\left(\mathrm{R}^{2}\right)$ obtidos a partir das análises de regressão entre os valores medidos e estimados da radiação líquida em Araras-SP por meio dos modelos de Zanetti (MZA) e Fietz e Fisch (MFF).

Tabela 2. Valores do coeficiente angular do modelo (b) e do coeficiente de determinação $\left(\mathrm{R}^{2}\right)$ utilizados na avaliação do desempenho dos modelos de Zanetti (MZA) e Fietz e Fisch (MFF) na estimativa da radiação líquida $(\mathrm{Rn})$ em Araras-SP.

\begin{tabular}{lcccccccc}
\hline \multirow{2}{*}{ Modelo } & \multicolumn{2}{c}{ Total } & \multicolumn{2}{c}{ Verão } & \multicolumn{2}{c}{ Inverno } & \multicolumn{2}{c}{ Média } \\
\cline { 2 - 9 } & $\mathrm{b}$ & $\mathrm{R}^{2}$ & $\mathrm{~b}$ & $\mathrm{R}^{2}$ & $\mathrm{~b}$ & $\mathrm{R}^{2}$ & $\mathrm{~b}$ & $\mathrm{R}^{2}$ \\
\hline MZA & 0,74 & 0,63 & 0,89 & 0,48 & 0,73 & 0,59 & 0,79 & 0,57 \\
MFF & 1,00 & 0,63 & 1,00 & 0,54 & 0,93 & 0,60 & 0,98 & 0,59
\end{tabular}

Na Tabela 2 pode ser verificado que o MZA superestimou a Rn nos três grupamentos de dados meteorológicos estudados, enquanto que o MFF o fez no grupamento Inverno. Assim como o MHS, o MZA e o MFF também utilizam em sua estrutura matemática uma função potência da amplitude térmica, mas contrariamente ao que ocorreu com o MHS no caso da estimativa da Rs, eles superestimaram a Rn.

Quanto à exatidão das estimativas da $\mathrm{Rn}$, pode ser verificado que o MFF teve excelente desempenho nos três grupamentos de dados, visto que os coeficientes angulares das respectivas equações de regressão foram praticamente um. Embora no MZA, a semelhança da lei de Stefan-Boltzmann para as emissões eletromagnéticas, a amplitude térmica esteja elevada a uma potência próxima de quatro, esse modelo superestimou a Rn, em média, em 20\% (Tabela 2)

Quanto à precisão nas estimativas, pode ser verificado que ela foi praticamente a mesma para os dois modelos estudados, com ligeira vantagem para o MFF (Tabela 2)

Resumindo o que foi exposto, pode-se concluir que o MFF estima a Rn para a região de Araras-SP com alta exatidão, porém com média precisão. É importante destacar que no presente estudo a Rn medida esta relacionada com uma superfície gramada, com albedo entre 23-25\%.
No ajustamento dos modelos empíricos para a estimativa da Rs e da Rn para a região de Araras-SP foram utilizados os mesmos dados meteorológicos utilizados para testar os modelos empíricos disponíveis na literatura, bem como, também foram mantidos os mesmos grupamentos dos dados meteorológicos (Total, Inverno e Verão).

No ajuste dos modelos empíricos relativos às relações funcionais exposta nas Equações de (1) a (6) foram utilizadas as seguintes funções matemáticas: polinomial do primeiro (função linear) e do segundo grau (função quadrática), potência e exponencial.

Os melhores ajustes, considerando-se os grupamentos de dados meteorológicos Total, Inverno e Verão, foram obtidos com a função potência, tanto para a estimativa da Rs (Figura 1), quanto para a estimativa Rn (Figura 2). Como segunda opção, com indicadores estatísticos ligeiramente inferiores aos obtidos com a função potência, ficou o modelo baseado em uma polinomial do segundo grau, enquanto que os modelos fundamentados na função linear e na função exponencial tiveram desempenho bem menos satisfatório.

Pela Figura 1, pode ser verificado que, no que se refere à exatidão dos modelos, o modelo baseado na função potência da amplitude térmica estimou a Rs com excelente exatidão nos três grupamentos de dados, com especial destaque para os grupamentos Inverno e total, 
AR ATMOSFÉRICO PARA A ESTIMATIVA DAS RADIAÇÕES GLOBAL E LÍQUIDA

ambos com erro médio de apenas $2 \%$. No caso do grupamento Verão, porém, o erro de estimativa foi mais alto, próximo de $9 \%$, o que certamente se deveu a uma maior presença de nuvens nesse período.

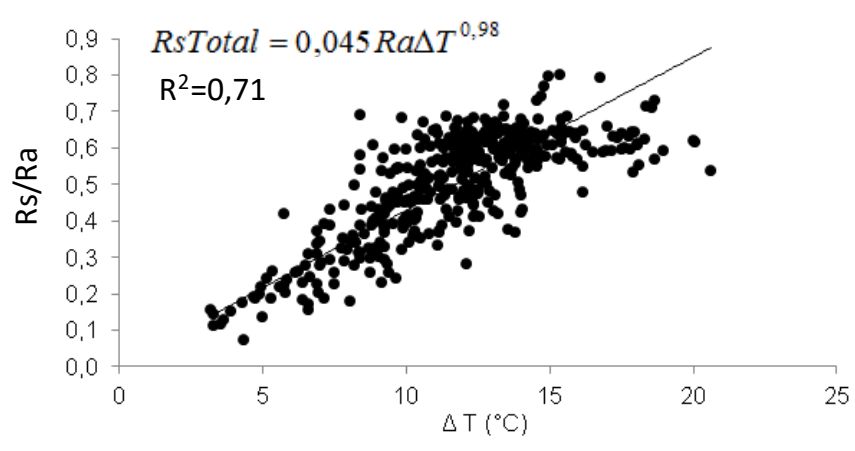

(a)

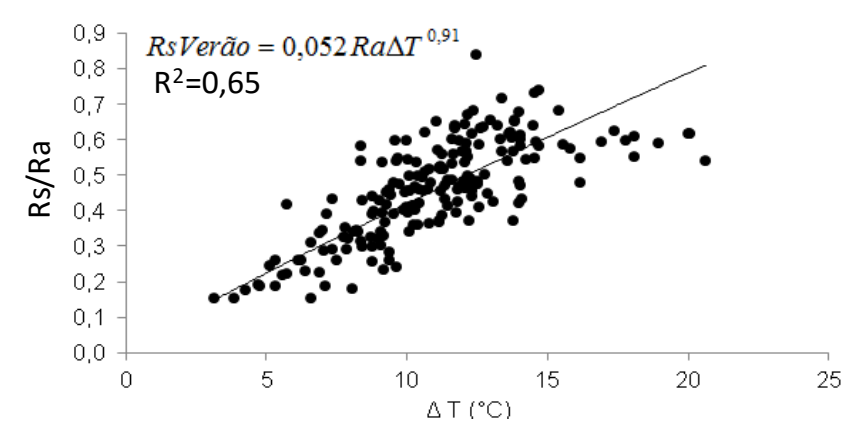

(b)

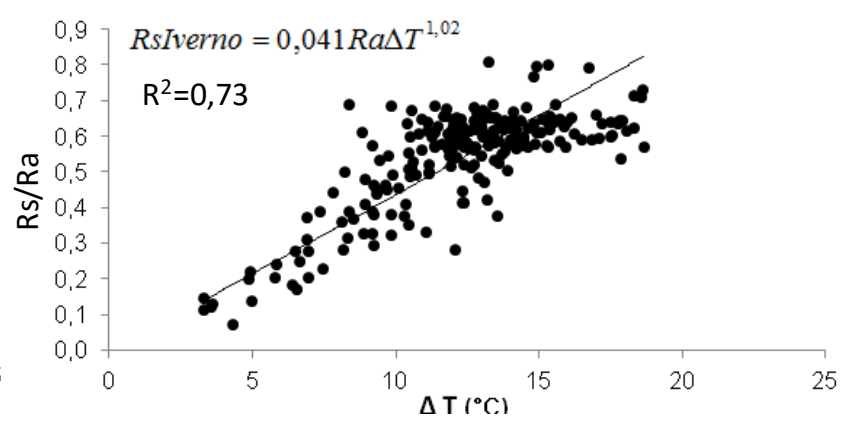

(c)

Figura 1. Modelos propostos para estimativa da radiação global (Rs) em Araras-SP, considerando-se três formas de grupamento dos dados meteorológicos: a) Total; b) Verão; c) Inverno.

Quanto à precisão do modelo, pode ser observado que o seu melhor desempenho se deu no grupamento Inverno, o que, pode, está condicionado a uma menor presença de nuvens nesse período.

Considerando-se os três grupamentos de dados definidos nesta pesquisa (Total, Inverno e Verão) a radiação global em ArarasSP pode ser estimada, com elevada exatidão e boa precisão, pelos modelos apresentados nas Equações 7 a 9:

$$
\begin{aligned}
& \text { RsTotal }=0,045 R a \Delta T^{0,98} \\
& \text { RsIverno }=0,041 R a \Delta T^{1,02} \\
& \text { RsVerão }=0,052 R a \Delta T^{0,91}
\end{aligned}
$$

na qual Rs é a radiação global $\left(\mathrm{MJ} \mathrm{m}^{-2} \mathrm{dia}^{-1}\right) ; \mathrm{Ra}$ a radiação extraterrestre $\left(\mathrm{MJ} \mathrm{m}^{-2} \mathrm{dia}^{-1}\right)$ e $\Delta \mathrm{T}$ a amplitude térmica diária do ar $\left({ }^{\circ} \mathrm{C}\right)$.

Pela Figura 2 pode ser verificado que a $\mathrm{Rn}$ foi estimada com uma exatidão menor do que a Rs pelo modelo potencial. Nela pode ser observado que os erros foram mais elevados no grupamento Total (11\%) e no Verão (18\%) do que no de Inverno (4\%), diferença esta que pode ser creditada a maior nebulosidade daqueles períodos. Outra explicação para este desempenho menos satisfatório do modelo potencial é que o balanço de radiação líquida é muito complexo, pois sofre a influência de outros parâmetros meteorológicos, como a umidade relativa e a velocidade do vento.

Assim como aconteceu com a exatidão das estimativas, o modelo potencial também apresentou uma precisão mais baixa na 
estimativa da radiação líquida comparativamente às estimativas da radiação global. Assim como ocorreu no caso da radiação global, o modelo potencial teve sua melhor precisão na estimativa da radiação líquida no grupamento Inverno.

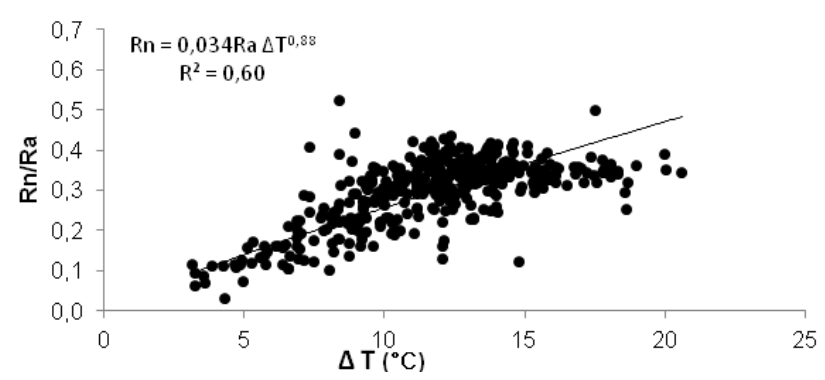

(a)

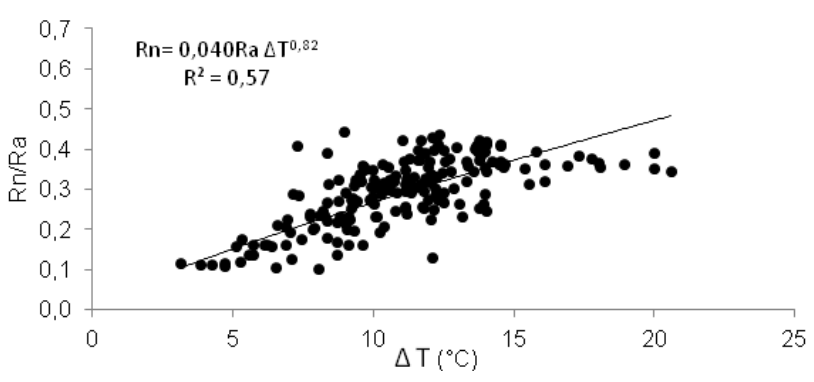

(b)

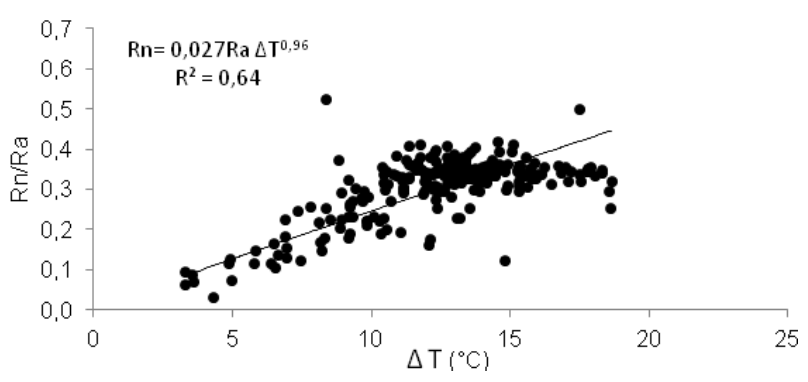

(c)

Figura 2. Modelos propostos para estimativa da radiação líquida (Rn) em Araras-SP, considerando-se três formas de grupamento dos dados meteorológicos: a) Total; b) Verão; c) Inverno.

Considerando-se os grupamentos de dados estabelecidos neste estudo (Total, Inverno e Verão) a Rn em Araras-SP pode ser estimada, com boa exatidão e precisão, pelos modelos apresentados nas Equações de 10 a 12:

$$
\begin{aligned}
& \text { RnTotal }=0,034 R a \Delta T^{0,89} \\
& \text { RnInverno }=0,027 \operatorname{Ra} \Delta T^{0,96} \\
& \text { RnVerão }=0,040 R a \Delta T^{0,82}
\end{aligned}
$$

na qual Rn é a radiação líquida ( $\left.\mathrm{MJ} \mathrm{m}^{-2} \mathrm{dia}^{-1}\right)$; $\mathrm{Ra}$ a radiação extraterrestre $\left(\mathrm{MJ} \mathrm{m}^{-2} \mathrm{dia}^{-1}\right)$ e $\Delta \mathrm{T}$ a amplitude térmica diária do ar $\left({ }^{\circ} \mathrm{C}\right)$.
O desempenho na estimativa da radiação global para a região de Araras-SP dos modelos disponíveis na literatura e os propostos nesta pesquisa está descrito na Tabela 3. Os modelos propostos estimaram a Rs com alta precisão, com um erro médio, para mais, de apenas 6,3\% nos três grupamentos de dados, enquanto no caso do MHS, MBC e MWE estes erros foram, respectivamente, de 17,7, 18,3 e 14,3\%. Quanto à precisão das estimativas, os modelos propostos estimaram radiação global nos três grupamentos de dados com precisão média alta $\left(\mathrm{R}^{2}=0,77\right)$, enquanto que no caso do MHS, MBC e MWE ela foi mais baixa, respectivamente, de 0,59 , 0,64 e 0,59 .

Tabela 3. Valores do coeficiente angular do modelo (b) e do coeficiente de determinação $\left(\mathrm{R}^{2}\right)$ dos modelos de Hargreaves e Samani (MHS), Bristow e Campbell (MBC), Weiss (MWE) e dos modelos propostos na estimativa da radiação global (Rs) em Araras-SP.

\begin{tabular}{ccccccc}
\multirow{2}{*}{ Modelo } & \multicolumn{2}{c}{ Total } & \multicolumn{2}{c}{ Verão } & \multicolumn{2}{c}{ Inverno } \\
\cline { 2 - 7 } & $\mathrm{b}$ & $\mathrm{R}^{2}$ & $\mathrm{~b}$ & $\mathrm{R}^{2}$ & $\mathrm{~b}$ & $\mathrm{R}^{2}$ \\
\hline \multirow{2}{*}{ MHS } & 0,96 & 0,57 & 1,30 & 0,54 & 1,19 & 0,66
\end{tabular}




\begin{tabular}{ccccccc} 
MBC & 0,81 & 0,61 & 0,71 & 0,62 & 0,93 & 0,69 \\
MWE & 0,85 & 0,55 & 0,76 & 0,53 & 0,96 & 0,69 \\
Proposto & 0,94 & 0,81 & 0,96 & 0,70 & 0,91 & 0,81 \\
\hline
\end{tabular}

O desempenho na estimativa da Rn para a região de Araras-SP dos modelos disponíveis na literatura e os aqui propostos estão apresentados na Tabela 4. Nela pode ser verificado que os modelos propostos estimaram a radiação líquida com alta precisão nos grupamentos Total e Verão, com erros, para mais, de, respectivamente, um e $5 \%$, o mesmo não acontecendo no grupamento
Inverno onde o erro, para mais, foi de $15 \%$, resultado este esperado em razão da menor presença de nuvens nesta estação do ano. Considerando-se os três grupamentos de dados, é importante destacar que o MFF (erro médio para mais de 2\%) teve desempenho superior que os modelos propostos (erro médio para mais de 7\%) especificamente para a região de Araras-SP.

Tabela 4. Valores do coeficiente angular do modelo (b) e do coeficiente de determinação $\left(\mathrm{R}^{2}\right)$ dos modelos de Zanetti (MZA), Fietz e Fisch (MFF) e dos modelos propostos na estimativa da radiação líquida (Rn) em Araras-SP.

\begin{tabular}{lcccccc}
\cline { 2 - 7 } \multicolumn{1}{c}{ Modelo } & \multicolumn{2}{c}{ Total } & \multicolumn{2}{c}{ Verão } & \multicolumn{2}{c}{ Inverno } \\
\cline { 2 - 7 } & $\mathrm{b}$ & $\mathrm{R}^{2}$ & $\mathrm{~b}$ & $\mathrm{R}^{2}$ & $\mathrm{~b}$ & $\mathrm{R}^{2}$ \\
\hline MZA & 0,74 & 0,63 & 0,89 & 0,48 & 0,73 & 0,59 \\
MFF & 1 & 0,63 & 1 & 0,54 & 0,93 & 0,60 \\
Proposto & 0,99 & 0,81 & 0,95 & 0,67 & 0,85 & 0,76 \\
\hline
\end{tabular}

Pelo que foi exposto e discutido anteriormente, pode-se afirmar que, na falta de equipamentos específicos para a medição da radiação global e da radiação líquida em ArarasSP, elas podem ser estimadas adequadamente utilizando-se apenas os valores medidos das temperaturas máxima e mínima diárias do ar atmosférico.

\section{CONCLUSÕES}

Considerando-se as características climáticas de Araras-SP, os resultados obtidos nesta pesquisa permitem concluir que:

1) A radiação global pode ser estimada com exatidão e precisão comparáveis pelos modelos disponíveis na literatura de Hargreaves e Samani, Bristow e Campbell e Weiss.

2) A radiação líquida para uma superfície gramada pode ser estimada com exatidão pelo modelo de Fietz e Fisch, também disponível na literatura especializada.
3) A radiação global (Rs) e a radiação líquida (Rn) diárias para a região de Araras-SP podem ser estimadas com precisão e exatidão adequadas pelos modelos propostos neste estudo.

\section{REFERÊNCIAS BIBLIOGRÁFICAS}

ALLEN, R.G.; PEREIRA, L.; RAES, D.; SMITH, M. Crop evapotranspiration: guidelines for computing crop water requirements. Rome: FAO, 1998. (FAO. Irrigation and Drainage Paper, 56).

AL-MOSTAFA, Z. A; MAGHRABI, A. H.; AL-SHEHRI, S. M. Sunshine-based global radiation models: a review and case study. Energy Conversion and Management, v.84, p.209-16, 2014.

BADESCU V.; DUMITRESCU, A. New models to compute solar global hourly irradiation from point cloudiness. Energy Conversion and Management, v.67, p.75-91, 2013 
BENGHANEM, M.; MELLIT, A. A simplified calibrated model for estimating daily global solar radiation in Madinah, Saudi Arabia. Theorical and Applied Climatolology, v.115, n.1, p.197-205, 2014

BORGES, V. P.; OLIVEIRA, A. S. de.; COELHO FILHO, M. A.; DA SILVA, T. S. M. PAMPONET, B. M. Avaliação de modelos de estimativa da radiação solar incidente em Cruz das Almas, Bahia. Revista Brasileira de Engenharia Agrícola e Ambiental, v.14, n.1, p.74-80, 2010

BRISTOW, K. L.; CAMPBELL, G. S. On the relationship between incoming solar radiation and daily maximum and minimum temperature. Agricultural and Forest Meteorology, v.31, n.2, p.159-166, 1984.

BURMAN, R.; POCHOP, L. O. Evaporation, evapotranspiration and climatic data. Amsterdan: Elsevier, 1994. 278p.

CONCEIÇÃO, M. A. F. Novo método para estimativa da evapotranspiração de referência com base na temperatura do ar. Bento Gonçalves: Embrapa Uva e Vinho, 2009. (Embrapa Uva e Vinho. Comunicado Técnico 97).

CONCEIÇÃO, M. A. F.; MANDELLI, F. Comparação entre métodos de estimativa da evapotranspiração de referência em Bento Gonçalves-RS. Revista Brasileira de Agrometeorologia, v.13, n.2, p.303-307, 2005.

CONCEIÇÃO, M. A. F.; MARIN, F. R. Avaliação de modelos para a estimativa de valores diários da radiação solar global com base na temperatura do ar. Revista Brasileira de Agrometeorologia, v.15, n.1, p.103-108, 2007.

DESPOTOVIC M.; NEDIC, V.; DESPOTOVIC, D.; CVETANOVIC, S. Review and statistical analysis of different global solar radiation sunshine models. Renewable and Sustainable Energy Reviews, v.52, p.18691880, 2015
DOORENBOS, J.; PRUITT, W. O. Guidelines for prediction crop water requirements. Rome: FAO: Irrigation and Drainage Paper 24. 1977

FIETZ, C. R.; FISCH, G. F. Avaliação de modelos de estimativa do saldo de radiação e do método de Priestley-Taylor para a região de Dourados, MS. Revista Brasileira Engenharia Agrícola Ambiental. v.13, n.4, 2009.

GOODIN, D. G; HUTCHINSON, J. M. S.; VANDERLIP, R. L.; KNAPP, M. C. Estimating solar irradiance for crop modeling using daily air temperature data. Agronomy Journal, v.91, n.5, p.845-51, 1999.

HARGREAVES, G. H.; SAMANI, Z. A. Estimating potential evapotranspiration. Journal of Irrigation and Drainage Engineering, v.108, p.225-230, 1982.

KHATIB, T., MOHAMED, A., SOPIAN, K. A review of solar energy modeling techniques. Renewable and Sustainable Energy Reviews, v.16, p.2864-2869, 2012.

KIRMANI, S.; JAMIL, M.; RIZWAN, M. Empirical correlation of estimating global solar radiation using meteorological parameters. International Journal of Sustainable Energy, v.34, p.327-39, 2015.

LIU D. L; SCOTT B. J. Estimation of solar radiation in Australia from rainfall and temperature observations. Agricultural and Forest Meteorology, v.106, n.1, p.41-59, 2001

LIU, X.; MEI, X.; LI, Y.; WANG, Q.; JENSEN, J. R.; ZHANG, Y.; PORTER, J. R. Evaluation of temperature- based global solar radiation models in China. Agricultural and Forest Meteorology, v.149, n.9, p.1433-46, 2009.

MONTONE, V. O.; SENTELHAS, P. C. Modelos de estimativa do saldo horário de radiação (Rn) para Piracicaba- SP. In: CONGRESSO BRASILEIRO DE METEOROLOGIA, 16, 2010. Belém-PA: 
Anais... Belém-PA: Sociedade Brasileira de Meteorologia. 1 CD.

PEREIRA, A. R.; ANGELOCCI, L. R., SENTELHAS, P. C. Agrometeorologia: Fundamentos e aplicações práticas. GuaíbaRS: Editora Agropecuária, 2002. 478p.

QUEJ, V. H.; ALMOROX, J.; IBRAKHIMOV, M.; SAITO, L. Empirical models for estimating daily global solar radiation in Yucatán Peninsula, Mexico. Energy Conversion and Management, v.110, p.448-456, 2016.

SANTOS, C.M.; SOUZA, J. L.; FERREIRAJUNIOR, R. A.; TIBA, C.; MELO, R. O.; LYRA, G. B.; TEODORO, I.; LYRA, G. B.; LEMES, M. A. M. On modeling global solar irradiation using air temperature for Alagoas State, Northeastern Brazil. Energy, v.71, p.38898, 2015.

SENTELHAS, P. C.; NASCIMENTO, A. L. C. Variação sazonal da relação entre o saldo de radiação e a irradiância solar global. Revista Brasileira de Meteorologia, v.18, n.1, 71-77, 2003.

WEISS, A.; HAYS, C. J.; HU, Q.; EASTERLING, W. E. Incorporating bias error in calculating solar irradiance: implications for crop simulations. Agronomy Journal, v.93, n.6, p.1321-1326, 2001.

YACEF, R.; MELLIT, A.; BELAID, S.; SEN, Z. New combined models for estimating daily global solar radiation from measured air temperature in semi-arid climates: application in Ghardaïa, Algeria. Energy Conversion Management, v.79, p.606-615, 2014.

ZANTTI, S. S.; SOUSA, E. F.; BERNARDO, S.; PSSE, R. P.; MELO JÚNIOR, J. C. F. Estimação do saldo de radiação e da evapotranspiração de referência, em função das temperaturas máxima e mínima do ar. Revista Brasileira de Agrometeorologia, v.14, n.1, p.134-139, 2006. 